\title{
Total Syntheses of Schulzeines B and C
}

Mukund K. Gurjar, Chinmoy Pramanik, Debabrata Bhattasali, C. V. Ramana and Debendra K.

\author{
Mohapatra
}

National Chemical Laboratory, Dr. Homi Bhabha Road, Pune - 411 008, India.

E-mail: mk.gurjar@ncl.res.in

*Corresponding author.Tel. : +91-20-25902627; fax: +91-20-25902629.

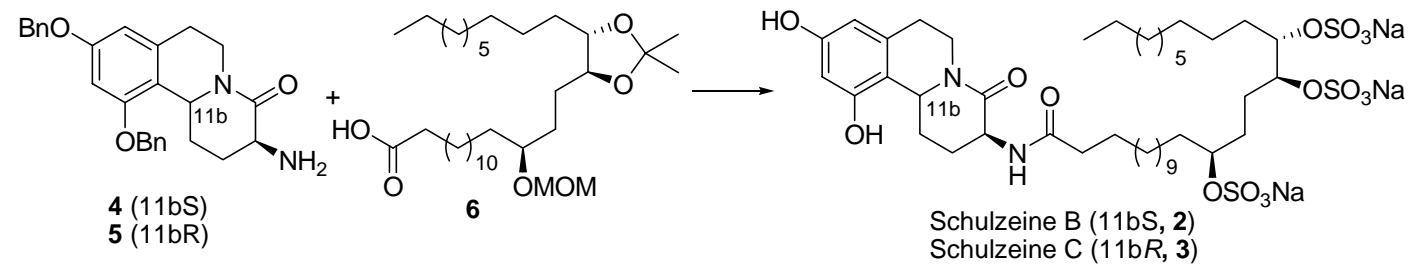


Table of Contents:

S. No. Description

1 General Methods

$2 \quad$ Entry 9

$\begin{array}{rr}\text { Synthesis } & \text { S4 } \\ \text { Spectral Data } & \text { S5 }\end{array}$

3

Entry 13

Synthesis S5

Spectral Data S6

$4 \quad$ Entry 14

Spectral Data S6

S7

$5 \quad$ Entry 17

$6 \quad$ Entry 18

Synthesis
Spectral Data

Synthesis S8

Spectral Data

Entry 21

$\begin{array}{rr}\text { Synthesis } & \text { S8 } \\ \text { Spectral Data } & \text { S9 }\end{array}$

$\begin{array}{ll}\text { Synthesis } & \text { S9 } \\ & \text { S10 }\end{array}$

$9 \quad$ Entry 23

$\begin{array}{rr}\text { Synthesis } & \text { S10 } \\ \text { Spectral Data } & \text { S11 }\end{array}$

$10 \quad$ Entry 24

$\begin{array}{rr}\text { Synthesis } & \text { S11 } \\ \text { Spectral Data } & \text { S12 }\end{array}$

$11 \quad$ Entry 25

Spectral Data $\quad$ S12

$12 \quad$ Entry 26

S4

5

5

S6

\section{Page}


$13 \quad$ Entry 27

$\begin{array}{rr}\text { Synthesis } & \text { S13 } \\ \text { Spectral Data } & \text { S14 }\end{array}$

$14 \quad$ Entry 28

$\begin{array}{rr}\text { Synthesis } & \text { S14 } \\ \text { Spectral Data } & \text { S15 }\end{array}$

$15 \quad$ Entry 29

$\begin{array}{ll}\text { Spectral Data } & \text { S15 } \\ & \text { S16 }\end{array}$

$16 \quad$ Entry 30

Spectral Data S16

S17

$17 \quad$ Entry 3

Spectral Data $\quad$ S17

S18 
General method: Air and/or moisture sensitive reactions were carried out in anhydrous solvents under an atmosphere of argon in an oven/flame-dried glassware. All anhydrous solvents were distilled prior to use: THF, benzene, toluene and diethyl ether from $\mathrm{Na}$ and benzophenone; $\mathrm{CH}_{2} \mathrm{Cl}_{2}, \mathrm{~N}-$ methyl pyrolidinone from $\mathrm{CaH}_{2} ; \mathrm{MeOH}$, EtOH from $\mathrm{Mg}$ cake. Commercial reagents were used without purification. Column chromatography was carried out by using Spectrochem silica gel (60-120 mesh). Specific optical rotations $[\alpha]_{\mathrm{D}}$ are given in $10^{-1} \mathrm{degcm}^{2} \mathrm{~g}^{-1}$. Infrared spectra were recorded in $\mathrm{CHCl}_{3} /$ neat (as mentioned) and reported in wave number $\left(\mathrm{cm}^{-1}\right) .{ }^{1} \mathrm{H}$ and ${ }^{13} \mathrm{C} \mathrm{NMR}$ chemical shifts are reported in ppm downfield from tetramethylsilane and coupling constants $(J)$ are reported in hertz $(\mathrm{Hz})$. The following abbreviations are used to designate signal multiplicity: $\mathrm{s}=$ singlet, $\mathrm{d}=$ doublet, $\mathrm{t}=$ triplet, $\mathrm{q}=$ quartet, $\mathrm{m}=$ multiplet, $\mathrm{br}=$ broad.

\section{(S)-Methyl-2-(benzyloxycarbonylamino)-5-(3,5-bis(benzyloxy)phenethylamino)-5-oxopentanoate (9).}

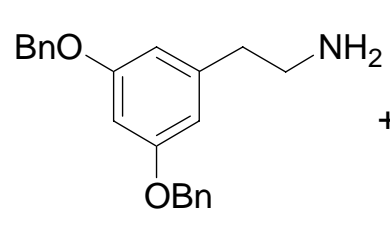

7

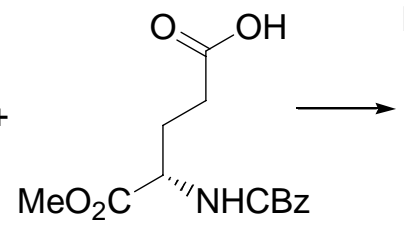

8

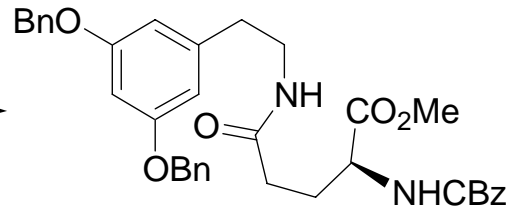

9

To a solution of $8(8.5 \mathrm{~g}, 29.4 \mathrm{mmol})$ in dry dichloromethane $(100 \mathrm{~mL})$ at $0{ }^{\circ} \mathrm{C}$ under nitrogen, EDC (6.77 g, $35.3 \mathrm{mmol})$, HOBt (4.76 g, $35.3 \mathrm{mmol})$ and compound 7 (8.83 g, $26.5 \mathrm{mmol})$ in dry dichloromethane $(50 \mathrm{~mL})$ were added. After stirring at room temperature for $10 \mathrm{~h}$, the reaction mixture was diluted with water, extracted with dichloromethane $(100 \mathrm{~mL} \times 2)$. The combined organic layer was washed with water, dried over anhydrous $\mathrm{Na}_{2} \mathrm{SO}_{4}$, concentrated and the residue chromatographed on silica gel by using ethyl acetate and light petroleum (2:3) to obtain $9(15.1 \mathrm{~g}, 84 \%)$ as a white solid. $\operatorname{mp} .114{ }^{\circ} \mathrm{C} ;[\alpha]_{\mathrm{D}}=-3.5\left(c 1.1, \mathrm{CHCl}_{3}\right) ; \mathrm{IR}\left(\mathrm{CHCl}_{3}\right) v_{\max }\left(\mathrm{cm}^{-1}\right) 3325,3032,2950,1719,1654,1593$, 1528, 1453, 1157, 1050, 753; ${ }^{1} \mathrm{H}$ NMR $\left(200 \mathrm{MHz}, \mathrm{CDCl}_{3}\right): \delta 7.43-7.31(\mathrm{~m}, 15 \mathrm{H}), 6.50(\mathrm{t}, J=2.2 \mathrm{~Hz}$, $1 \mathrm{H}), 6.45(\mathrm{~d}, J=2.2 \mathrm{~Hz}, 2 \mathrm{H}), 5.83(\mathrm{t}, J=6.2 \mathrm{~Hz}, 1 \mathrm{H}), 5.69(\mathrm{~d}, J=8.0 \mathrm{~Hz}, 1 \mathrm{H}), 5.08(\mathrm{~s}, 2 \mathrm{H}), 5.01(\mathrm{~s}$, 
4H), $4.31(\mathrm{~m}, 1 \mathrm{H}), 3.71(\mathrm{~s}, 3 \mathrm{H}), 3.48(\mathrm{dt}, J=6.9,6.2 \mathrm{~Hz}, 2 \mathrm{H}), 2.73(\mathrm{t}, J=6.9 \mathrm{~Hz}, 2 \mathrm{H}), 2.18(\mathrm{~m}, 2 \mathrm{H})$, $1.96(\mathrm{~m}, 1 \mathrm{H}), 1.66(\mathrm{~m}, 1 \mathrm{H}) ;{ }^{13} \mathrm{C}\left(50 \mathrm{MHz}, \mathrm{CDCl}_{3}\right): \delta 172.3,171.6,160.0,156.2,141.1,136.7,136.1$, $128.4,128.4,128.2,128.0,127.9,127.8,127.4,107.8,100.0,69.9,66.9,53.4,52.3,40.3,35.7,32.2$, 28.2; MS (ESI) m/z: Calcd for $\left(\mathrm{M}^{+}+\mathrm{Na}\right)$ 633.27. Found: 633.42; Anal. Calcd for $\mathrm{C}_{36} \mathrm{H}_{38} \mathrm{~N}_{2} \mathrm{O}_{7}$ : $\mathrm{C}, 70.80$; H, 6.27; N, 4.59. Found: C, 70.8; H, 6.22; N, 4.46.

tert-Butyl-(3S,11bS)-9,11-bis(benzyloxy)-4-oxo-2,3,4,6,7,11b-hexahydro-1H-pyrido[2,1a]isoquino- lin-3-ylcarbamate (13).

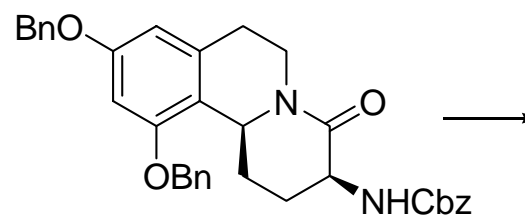

11

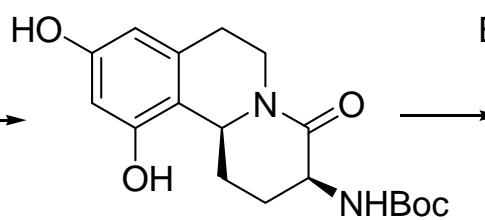

NHBoc<smiles>CC(C)(C)OCc1cccc(OCc2ccccc2)c1</smiles>

13

A suspension of $11(1.8 \mathrm{~g}, 3.2 \mathrm{mmol})$ and 10\% $\mathrm{Pd} / \mathrm{C}(100 \mathrm{mg})$ in methanol (15 mL) was hydrogenated at normal pressure and temperature for $5 \mathrm{~h}$. $\mathrm{Boc}_{2} \mathrm{O}(1.0 \mathrm{~mL}, 4.16 \mathrm{mmol})$ was added to the reaction mixture and stirred for $5 \mathrm{~h}$ at room temperature. The catalyst was removed by filtration through a pad of Celite and the filtrate concentrated. The residue was purified on silica gel by eluting with ethyl acetate and light petroleum (1.5:3.5) to afford tert-butyl (3S,11bS)-9,11-dihydroxy-4-oxo-2,3,4,6,7,11bhexahydro-1H-pyrido[2,1-a]isoquinolin-3-ylcarbamate $(1.08 \mathrm{~g}, 96 \%)$ as a gummy paste. $[\alpha]_{\mathrm{D}}=-49.1(\mathrm{c}$ $0.9, \mathrm{CH}_{3} \mathrm{OH}$ ); IR (nujol) $v_{\max }\left(\mathrm{cm}^{-1}\right) 3274,2924,2854,1683,1646,1511,1464,1376,1277,1251,1159$, 1055, 947, 842; ${ }^{1} \mathrm{H}$ NMR (200 MHz, $\left.\mathrm{CD}_{3} \mathrm{OD}\right): \delta 6.18(\mathrm{~d}, J=2.3 \mathrm{~Hz}, 1 \mathrm{H}), 6.11(\mathrm{~d}, J=2.3 \mathrm{~Hz}, 1 \mathrm{H}), 4.81$ $(\mathrm{dd}, J=11.2,3.8 \mathrm{~Hz}, 1 \mathrm{H}), 4.56(\mathrm{~m}, 1 \mathrm{H}), 4.31(\mathrm{dd}, J=10.6,7.3 \mathrm{~Hz}, 1 \mathrm{H}), 2.70-2.27(\mathrm{~m}, 5 \mathrm{H}), 1.56-1.49$ $(\mathrm{m}, 1 \mathrm{H}), 1.46(\mathrm{~s}, 9 \mathrm{H}), 1.37-1.31(\mathrm{~m}, 1 \mathrm{H}) ;{ }^{13} \mathrm{C}\left(50 \mathrm{MHz}, \mathrm{CD}_{3} \mathrm{OD}\right): \delta 172.3,157.8,156.0,138.3,115.0$ 107.2, 101.9, 80.5, 51.0, 50.7, 40.3, 30.2, 29.1, 28.7; MS (ESI) m/z: Calcd for $\left(\mathrm{M}^{+}+\mathrm{Na}\right) 371.17$. Found: 371.64; Anal. Calcd for $\mathrm{C}_{18} \mathrm{H}_{24} \mathrm{~N}_{2} \mathrm{O}_{5}$ : C, 62.05; H, 6.94; N, 8.04. Found: C, 62.0; H, 6.74; N, 7.81. 
To a solution of the above diol $(840 \mathrm{mg}, 2.41 \mathrm{mmol}), \mathrm{Cs}_{2} \mathrm{CO}_{3}(2.36 \mathrm{~g}, 7.24 \mathrm{mmol})$, TBAI $(20 \mathrm{mg})$ in dry DMF $(5 \mathrm{~mL})$ was added benzyl bromide $(0.63 \mathrm{~mL}, 5.3 \mathrm{mmol})$ at $0{ }^{\circ} \mathrm{C}$. After $1 \mathrm{~h}$, the reaction was diluted with ice-water and extracted with ethyl acetate $(20 \mathrm{~mL} \times 3)$. The combined organic layer was washed with water, dried over anhydrous $\mathrm{Na}_{2} \mathrm{SO}_{4}$, concentrated and the residue purified on silica gel by eluting with ethyl acetate and light petroleum (1:3) to afford (3S,11bS)-13 (1.18 g, 92\%), as a white powder. mp. $118^{\circ} \mathrm{C} ;[\alpha]_{\mathrm{D}}=-102\left(\mathrm{c} 1.1, \mathrm{CHCl}_{3}\right) ; \mathrm{IR}\left(\mathrm{CHCl}_{3}\right) v_{\max }\left(\mathrm{cm}^{-1}\right) 3412,3018,2931,1707,1653$, 1609, 1497, 1442, 1367, 1147, 1060; ${ }^{1} \mathrm{H} \mathrm{NMR}\left(200 \mathrm{MHz}, \mathrm{CDCl}_{3}\right): \delta 7.35(\mathrm{~m}, 10 \mathrm{H}), 6.45(\mathrm{~d}, J=2.2$ Hz, 1H), 6.36 (d, $J=2.2 \mathrm{~Hz}, 1 \mathrm{H}), 5.75$ (d, $J=5.3 \mathrm{~Hz}, 1 \mathrm{H}), 5.05(\mathrm{~s}, 2 \mathrm{H}), 4.97(\mathrm{~s}, 2 \mathrm{H}), 4.89$ (dd, $J=10.3$, $4.0 \mathrm{~Hz}, 1 \mathrm{H}), 4.76-4.68(\mathrm{~m}, 1 \mathrm{H}), 4.30(\mathrm{~m}, 1 \mathrm{H}), 2.86-2.40(\mathrm{~m}, 5 \mathrm{H}), 1.46(\mathrm{~s}, 9 \mathrm{H}), 1.42-1.34(\mathrm{~m}, 2 \mathrm{H}) ;{ }^{13} \mathrm{C}$ $\left(50 \mathrm{MHz}, \mathrm{CDCl}_{3}\right): \delta 170.1,158.2,155.7,155.4,137.0,136.5,136.2,128.5,128.3,127.9,127.8,127.2$, 126.8, 117.1, 105.7, 98.8, 79.0, 69.9, 69.8, 49.5, 48.5, 38.6, 29.5, 29.4, 28.3, 28.2; MS (ESI) m/z: Calcd for $\left(\mathrm{M}^{+}+\mathrm{Na}\right)$ 551.26. Found: 551.91; Anal. Calcd for $\mathrm{C}_{32} \mathrm{H}_{36} \mathrm{~N}_{2} \mathrm{O}_{5}$ : C, 72.70; H, 6.86; N, 5.30. Found: C, 72.85; H, 6.98; N, 5. 17.

tert-Butyl-(3S,11bR)-9,11-bis(benzyloxy)-4-oxo-2,3,4,6,7,11b-hexahydro-1H-pyrido[2,1-a]isoquinolin-3-ylcarbamate (14).

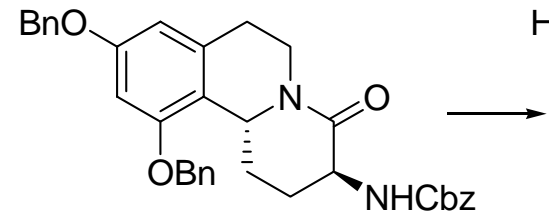

12

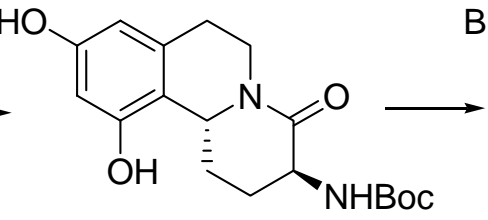

$12 a$

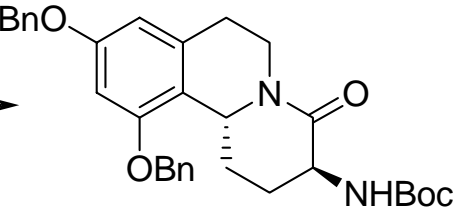

14

Compound 12 was transformed in to tert-butyl $(3 S, 11 \mathrm{~b} R)-9,11$-dihydroxy-4-oxo-2,3,4,6,7,11bhexahydro-1H-pyrido[2,1-a]isoquinolin-3-ylcarbamate (12a) (97\%) by using the same procedure as reported above. $[\alpha]_{\mathrm{D}}=+122\left(c\right.$ 1.4, $\left.\mathrm{CH}_{3} \mathrm{OH}\right)$; IR (nujol) $v_{\max }\left(\mathrm{cm}^{-1}\right) 3274,2924,2854,1683,1646,1511$, 1464, 1376, 1277, 1251, 1159, 1055, 947, 842; ${ }^{1} \mathrm{H}$ NMR $\left(200 \mathrm{MHz}, \mathrm{CD}_{3} \mathrm{OD}\right): \delta 6.16(\mathrm{~d}, J=1.9 \mathrm{~Hz}$, 1H), $6.08(\mathrm{~d}, J=1.9 \mathrm{~Hz}, 1 \mathrm{H}), 4.80(\mathrm{dd}, J=3.3 \mathrm{~Hz}, 1 \mathrm{H}), 4.74(\mathrm{dd}, J=2.8,1 \mathrm{H}), 3.96(\mathrm{~m}, 1 \mathrm{H}), 3.15-3.0$ $(\mathrm{m}, 1 \mathrm{H}), 2.72-2.48(\mathrm{~m}, 3 \mathrm{H}), 2.19-1.76(\mathrm{~m}, 3 \mathrm{H}), 1.45(\mathrm{~s}, 9 \mathrm{H}) ;{ }^{13} \mathrm{C}\left(50 \mathrm{MHz}, \mathrm{CD}_{3} \mathrm{OD}\right): \delta 171.0,159.6$, 157.6, 156.6, 138.6, 115.9, 107.6, 102.1, 57.2, 40.8, 31.0, 29.4, 28.7; MS (ESI) m/z: Calcd for $\left(\mathrm{M}^{+}+\right.$ 
Na) 371.17. Found: 371.61; Anal. Calcd for $\mathrm{C}_{18} \mathrm{H}_{24} \mathrm{~N}_{2} \mathrm{O}_{5}$ : C, 62.05; H, 6.94; N, 8.04. Found: C, 61.96;

H, 6.70; N, 7.85.

The resulting product was converted into $14(91 \%)$ as described above. $[\alpha]_{\mathrm{D}}=+116\left(\mathrm{c} 1.35, \mathrm{CHCl}_{3}\right)$; IR $\left(\mathrm{CHCl}_{3}\right) v_{\max }\left(\mathrm{cm}^{-1}\right) 3412,3018,2931,1707,1653,1609,1497,1442,1367,1147,1060 ;{ }^{1} \mathrm{H}$ NMR $\left(200 \mathrm{MHz}, \mathrm{CDCl}_{3}\right): \delta 7.42-7.34(\mathrm{~m}, 10 \mathrm{H}), 6.47(\mathrm{~d}, J=2.3 \mathrm{~Hz}, 1 \mathrm{H}), 6.35(\mathrm{~d}, J=2.3 \mathrm{~Hz}, 1 \mathrm{H}), 5.33(\mathrm{~d}, J=$ $5.0 \mathrm{~Hz}, 1 \mathrm{H}), 5.04-5.0(\mathrm{~m}, 4 \mathrm{H}), 4.96-4.88(\mathrm{~m}, 1 \mathrm{H}), 4.78(\mathrm{dd}, J=11.0,3.7 \mathrm{~Hz}, 1 \mathrm{H}), 3.99(\mathrm{~m}, 1 \mathrm{H}), 3.13-$ $2.78(\mathrm{~m}, 2 \mathrm{H}), 2.69-2.41(\mathrm{~m}, 3 \mathrm{H}), 1.83-1.64(\mathrm{~m}, 2 \mathrm{H}), 1.45(\mathrm{~s}, 9 \mathrm{H}) ;{ }^{13} \mathrm{C}\left(50 \mathrm{MHz}, \mathrm{CDCl}_{3}\right): \delta 168.7$, $158.1,156.7,156.1,137.8,136.6,136.4,128.6,128.5,128.0,127.4,127.1,118.3,106.0,99.0,79.4$, 70.1, 70.0, 56.1, 52.7, 39.4, 30.5, 29.6, 28.3, 27.9; MS (ESI) m/z: Calcd for $\left(\mathrm{M}^{+}+\mathrm{Na}\right)$ 551.26. Found: 551.91; Anal. Calcd for $\mathrm{C}_{32} \mathrm{H}_{36} \mathrm{~N}_{2} \mathrm{O}_{5}$ : C, 72.70; H, 6.86; N, 5.30. Found: C, 72.61; H, 6.77; N, 5.23.

\section{Methyl 14-(benzyloxy)tetradecanoate (17).}

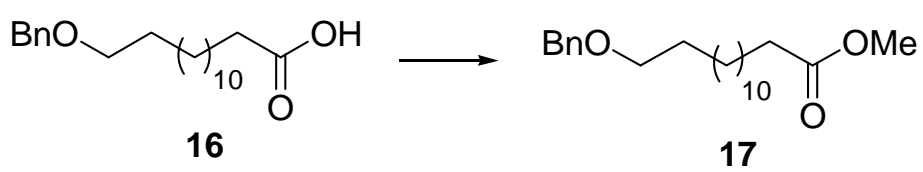

Compound 16 (4.6 g, $13.8 \mathrm{mmol})$, methanol (50 mL) and p-TSA (50 mg) were heated under reflux for 3 $\mathrm{h}$ and concentrated. The residue was extracted with ethyl acetate, washed with water, brine, dried over $\mathrm{Na}_{2} \mathrm{SO}_{4}$ and evaporated. The residue was purified on silica gel eluting with ethyl acetate and light petroleum $(0.5: 9.5)$ to afford $17(4.51 \mathrm{~g}, 94 \%)$ as colorless oil. IR $\left(\mathrm{CHCl}_{3}\right) v_{\max }\left(\mathrm{cm}^{-1}\right) 2927,2854$, 1738, 1602, 1495, 1363; ${ }^{1} \mathrm{H}$ NMR $\left(200 \mathrm{MHz}, \mathrm{CDCl}_{3}\right): \delta 7.33-7.25(\mathrm{~m}, 5 \mathrm{H}), 4.49(\mathrm{~s}, 2 \mathrm{H}), 3.66(\mathrm{~s}, 3 \mathrm{H})$, $3.45(\mathrm{t}, J=6.6 \mathrm{~Hz}, 2 \mathrm{H}), 2.29(\mathrm{t}, J=7.3 \mathrm{~Hz}, 2 \mathrm{H}), 1.64-1.54(\mathrm{~m}, 4 \mathrm{H}), 1.25(\mathrm{~m}, 18 \mathrm{H}) ;{ }^{13} \mathrm{C} \mathrm{NMR}(50 \mathrm{MHz}$, $\left.\mathrm{CDCl}_{3}\right): \delta 174.1,138.7,128.3,127.5,127.4,72.8,70.4,51.3,34.1,29.8,29.6,29.5(2 \mathrm{C}), 29.3,29.2$, 26.2, 24.9; MS (ESI) m/z: Calcd for $\left(\mathrm{M}^{+}+\mathrm{Na}\right)$ 371.27. Found: 371.26; Anal. Calcd for $\mathrm{C}_{22} \mathrm{H}_{36} \mathrm{O}_{3}$ : $\mathrm{C}_{\text {, }}$ 75.82; H, 10.41. Found: C, 75.62; H, 10.62. 


\section{Dimethyl 15-(benzyloxy)-2-oxopentadecylphosphonate (18).}

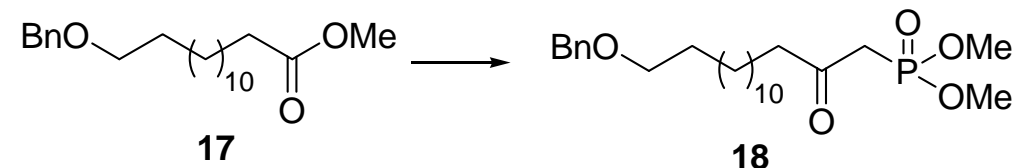

To a stirred solution of dimethylmethanephosphonate $(3.2 \mathrm{~g}, 25.9 \mathrm{mmol})$ in THF $(30 \mathrm{~mL})$ at $-78^{\circ} \mathrm{C}, n-$ BuLi (13.4 mL, 1.6 M in hexane, $21.5 \mathrm{mmol})$ was added. After $0.5 \mathrm{~h}, 17$ (3.0 g, $8.6 \mathrm{mmol})$ was introduced and stirring continued for $2.5 \mathrm{~h}$ at which time $10 \% \mathrm{Na}_{2} \mathrm{HPO}_{4}$ solution $(20 \mathrm{~mL})$ was added and layers separated. The organic layer was washed with water, the combined aqueous phase extracted with diethyl ether $(3 \times 50 \mathrm{~mL})$. The combined organic phase was dried over anhydrous $\mathrm{Na}_{2} \mathrm{SO}_{4}$ and evaporated. The residue was purified on silica gel by eluting with ethyl acetate and light petroleum (3:2) to afford $18(3.53 \mathrm{~g}, 93 \%)$ as yellow oil. IR $\left(\mathrm{CHCl}_{3}\right) v_{\max }\left(\mathrm{cm}^{-1}\right) 2927,2855,1715,1602,1454,1365$; ${ }^{1} \mathrm{H}$ NMR $\left(200 \mathrm{MHz}, \mathrm{CDCl}_{3}\right): \delta 7.33-7.25(\mathrm{~m}, 5 \mathrm{H}), 4.49(\mathrm{~s}, 2 \mathrm{H}), 3.81(\mathrm{~s}, 3 \mathrm{H}), 3.76(\mathrm{~s}, 3 \mathrm{H}), 3.45(\mathrm{t}, J=$ $6.6 \mathrm{~Hz}, 2 \mathrm{H}), 3.07(\mathrm{~d}, J=22.7 \mathrm{~Hz}, 2 \mathrm{H}), 2.60(\mathrm{t}, J=7.3 \mathrm{~Hz}, 2 \mathrm{H}), 1.67-1.54(\mathrm{~m}, 4 \mathrm{H}), 1.25(\mathrm{~m}, 18 \mathrm{H}) ;{ }^{13} \mathrm{C}$ NMR (50 MHz, $\left.\mathrm{CDCl}_{3}\right): \delta 201.7,201.5,138.6,128.2,127.4,127.3,72.7,70.3,52.9,44.0,42.4,39.9$, 29.7, 29.5, 29.4, 29.3, 28.8, 26.1, 23.2; MS (ESI) m/z: Calcd for $\left(\mathrm{M}^{+}+\mathrm{Na}\right)$ 463.27. Found: 463.25; Anal. Calcd for $\mathrm{C}_{24} \mathrm{H}_{41} \mathrm{O}_{5} \mathrm{P}: \mathrm{C}, 65.43 ; \mathrm{H}, 9.38$. Found: C, 65.26; H, 9.42.

\section{(2S,3R)-Ethyl 2,3-dihydroxytridecanoate (21).}

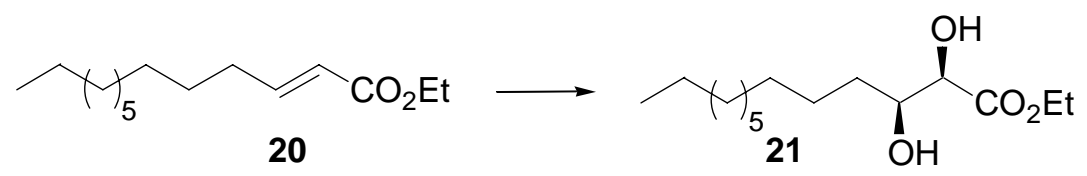

To a vigorously stirring mixture of $\mathrm{K}_{3}\left[\mathrm{Fe}(\mathrm{CN})_{6}\right](20.6 \mathrm{~g}, 62.5 \mathrm{mmol}), \mathrm{K}_{2} \mathrm{CO}_{3}(8.64 \mathrm{~g}, 62.5 \mathrm{mmol})$, (DHQ) ${ }_{2}$ PHAL (160 mg, $\left.0.2 \mathrm{mmol}\right), \mathrm{MeSO}_{2} \mathrm{NH}_{2}(1.98 \mathrm{~g}, 20.8 \mathrm{mmol})$ and $\mathrm{K}_{2} \mathrm{OsO}_{4} .2 \mathrm{H}_{2} \mathrm{O}(40 \mathrm{mg}, 0.1$ mmol) in (1:1) ${ }^{\mathrm{t}} \mathrm{BuOH}: \mathrm{H}_{2} \mathrm{O}(200 \mathrm{~mL})$ at $0{ }^{\circ} \mathrm{C}$ was added compound 20 (5.0 g, $\left.20.8 \mathrm{mmol}\right)$. After $6 \mathrm{~h}$, 
sodium sulphite $(10 \mathrm{~g})$ was added, tert. $\mathrm{BuOH}$ evaporated and the aqueous phase was extracted with ethyl acetate, washed with $2 \mathrm{~N} \mathrm{KOH}$, water, brine, dried over anhydrous $\mathrm{Na}_{2} \mathrm{SO}_{4}$ and concentrated. The residue was purified on silica gel by eluting with ethyl acetate and light petroleum (1:4) to afford 21 $(4.85 \mathrm{~g}, 85 \%)$ as a white solid. $\mathrm{mp} 62{ }^{\circ} \mathrm{C} ;[\alpha]_{\mathrm{D}}=-10.6\left(c 1.0, \mathrm{CHCl}_{3}\right) ; \mathrm{IR}\left(\mathrm{CHCl}_{3}\right) v_{\max }\left(\mathrm{cm}^{-1}\right) 3456$, 3018, 2927, 1734, 1466, 1370; ${ }^{1} \mathrm{H}$ NMR (200 MHz, $\left.\mathrm{CDCl}_{3}\right): \delta 4.29$ (q, $\left.J=7.1 \mathrm{~Hz}, 2 \mathrm{H}\right), 4.06(\mathrm{~d}, J=1.8$ Hz, 1H), 3.86 (dt, $J=6.3,1.8 \mathrm{~Hz}, 1 \mathrm{H}), 3.25$ (br. s, 1H), 2.25 (br. s, 1H), 1.62-1.55 (m, 2H), 1.36-1.27 $(\mathrm{m}, 19 \mathrm{H}), 0.88(\mathrm{t}, J=6.7 \mathrm{~Hz}, 3 \mathrm{H}) ;{ }^{13} \mathrm{C} \mathrm{NMR}\left(50 \mathrm{MHz}, \mathrm{CDCl}_{3}\right): \delta 173.6,73.2,72.5,61.6,33.5,31.8$, 29.5, 29.3, 25.7, 22.6, 14.0; MS (ESI) m/z: Calcd for $\left(\mathrm{M}^{+}+\mathrm{Na}\right)$ 297.21. Found: 297.20; Anal. Calcd for $\mathrm{C}_{15} \mathrm{H}_{30} \mathrm{O}_{4}$ : C, 65.66; H, 11.02. Found: C, 65.79; H, 11.07. Enantiomeric purity of 21 was estimated (98.5\% ee) by HPLC analysis of its dibenzoate derivative using a chiracel OD-H column $(0.8 \% \mathrm{i}-\mathrm{PrOH} /$ n-Hexane, flow rate $0.5 \mathrm{~mL} / \mathrm{min}, \lambda=225 \mathrm{~nm}$ ).

\section{(4R,5S)-5-Decyl-2,2-dimethyl-1,3-dioxolane-4-carbaldehyde (22).}

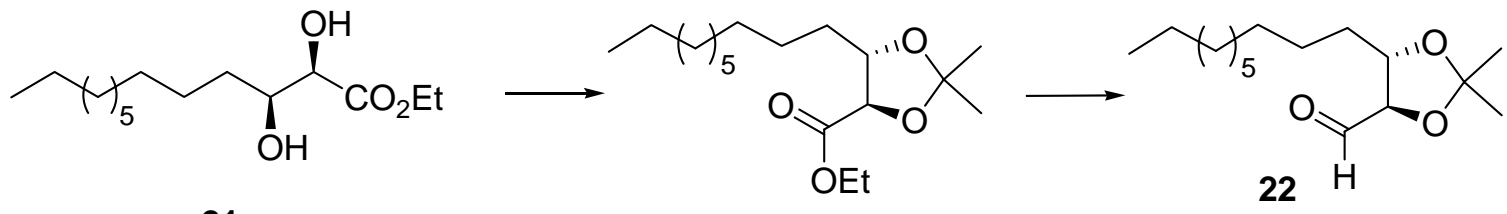

21

A solution of 21 (3.0 g, $10.9 \mathrm{mmol}), 2$,2-dimethoxypropane (1.6 mL, $13.1 \mathrm{mmol})$ and catalytic $p$-TSA in $\mathrm{CH}_{2} \mathrm{Cl}_{2}(30 \mathrm{~mL})$ were stirred at room temperature for $1 \mathrm{~h}$, washed with $\mathrm{NaHCO}_{3}$, water, brine, dried over anhydrous $\mathrm{Na}_{2} \mathrm{SO}_{4}$ and concentrated. The residue was purified on silica gel by using ethyl acetate and light petroleum $(0.5: 9.5)$ to afford the acetonide derivative $(3.27 \mathrm{~g}, 95 \%)$ as colorless oil. $[\alpha]_{\mathrm{D}}=-$ $15.4\left(\right.$ c 1.0, $\left.\mathrm{CHCl}_{3}\right)$; IR $\left(\mathrm{CHCl}_{3}\right) v_{\max }\left(\mathrm{cm}^{-1}\right)$ 2926, 2855, 1761, 1380; ${ }^{1} \mathrm{H}$ NMR (200 MHz, $\left.\mathrm{CDCl}_{3}\right): \delta$ $4.24(\mathrm{q}, J=7.1 \mathrm{~Hz}, 2 \mathrm{H}), 4.11-4.07(\mathrm{~m}, 2 \mathrm{H}), 1.77-1.65(\mathrm{~m}, 2 \mathrm{H}), 1.46(\mathrm{~s}, 3 \mathrm{H}), 1.43(\mathrm{~s}, 3 \mathrm{H}), 1.34-1.27$ $(\mathrm{m}, 19 \mathrm{H}), 0.88(\mathrm{t}, J=6.7 \mathrm{~Hz}, 3 \mathrm{H}) ;{ }^{13} \mathrm{C} \mathrm{NMR}\left(50 \mathrm{MHz}, \mathrm{CDCl}_{3}\right): \delta 170.8,110.6,79.1,61.1,33.5,31.8$, 29.5, 29.3, 27.1, 25.6, 22.6, 14.1; MS (ESI) m/z: Calcd for $\left(\mathrm{M}^{+}+\mathrm{Na}\right)$ 337.25. Found: 337.24; Anal. Calcd for $\mathrm{C}_{18} \mathrm{H}_{34} \mathrm{O}_{4}$ : C, 68.75; H, 10.90. Found: C, 68.64; H, 10.75. 
To a solution of above acetonide $(2.5 \mathrm{~g}, 7.9 \mathrm{mmol})$ in $\mathrm{CH}_{2} \mathrm{Cl}_{2}(30 \mathrm{~mL})$ at $-78{ }^{\circ} \mathrm{C}$ was added DIBAL-H (1.3 M solution in toluene, $7.30 \mathrm{~mL}, 9.5 \mathrm{mmol}$ ). After $1 \mathrm{~h}$, excess of DIBAL-H was quenched with saturated solution of sodium potassium tartrate. The solid was filtered, the filtrate concentrated to give a residue which was purified on silica gel by eluting with ethyl acetate and light petroleum (1:9) to give $22(2.0 \mathrm{~g}, 93 \%)$ as a colorless oil. Compound 22 was found to decompose on standing and therefore used for the next reaction immediately.

\section{(E)-16-(benzyloxy)-1-((4S,5S)-5-decyl-2,2-dimethyl-1,3-dioxolan-4-yl)hexadec-1-en-3-one (23).}

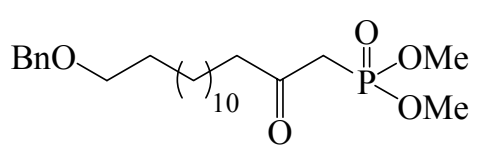

18

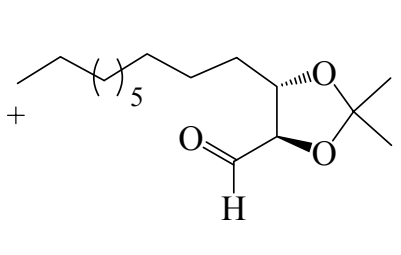

22

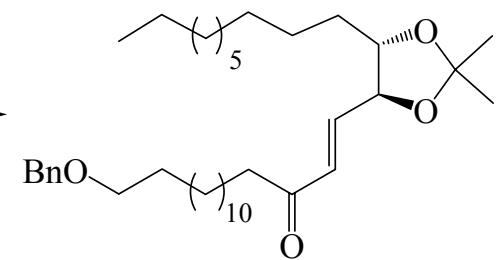

23

To a stirred solution of 18 (1.95 g, $4.4 \mathrm{mmol})$, DBU (560 mg, $3.7 \mathrm{mmol})$, and LiCl (190 mg, $4.4 \mathrm{mmol})$ in dry $\mathrm{CH}_{3} \mathrm{CN}(15 \mathrm{~mL})$ under nitrogen at room temperature was added $22(1.0 \mathrm{~g}, 3.69 \mathrm{mmol})$. After $1 \mathrm{~h}$, saturated $\mathrm{NH}_{4} \mathrm{Cl}$ solution was added, and the mixture was extracted with ether $(2 \times 15 \mathrm{~mL})$, washed with brine, dried over anhydrous $\mathrm{Na}_{2} \mathrm{SO}_{4}$ and evaporated. The residue was purified on silica gel by eluting with ethyl acetate and light petroleum (0.5:9.5) to afford $23(2.05 \mathrm{~g}, 95 \%)$ as a yellow oil. $[\alpha]_{\mathrm{D}}$ $=-6.1\left(c 1.3, \mathrm{CHCl}_{3}\right) ; \mathrm{IR}\left(\mathrm{CHCl}_{3}\right) v_{\max }\left(\mathrm{cm}^{-1}\right) 2855,2928,1697,1676,1495,1371 ;{ }^{1} \mathrm{H} \mathrm{NMR}(200 \mathrm{MHz}$, $\left.\mathrm{CDCl}_{3}\right): \delta 7.34-7.26(\mathrm{~m}, 5 \mathrm{H}), 6.70(\mathrm{dd}, J=15.8,5.7 \mathrm{~Hz}, 1 \mathrm{H}), 6.36(\mathrm{dd}, J=15.8,1.3 \mathrm{~Hz}, 1 \mathrm{H}), 4.49(\mathrm{~s}$, 2H), 4.17-4.09 (m, 1H), 3.76-3.66 (m, 1H), $3.45(\mathrm{t}, J=6.6 \mathrm{~Hz}, 2 \mathrm{H}), 2.55(\mathrm{t}, J=7.2 \mathrm{~Hz}, 2 \mathrm{H}), 1.64-1.51$ $(\mathrm{m}, 8 \mathrm{H}), 1.43(\mathrm{~s}, 3 \mathrm{H}), 1.38(\mathrm{~s}, 3 \mathrm{H}), 1.26(\mathrm{~m}, 32 \mathrm{H}), 0.88(\mathrm{t}, \mathrm{J}=6.7 \mathrm{~Hz}, 3 \mathrm{H}) ;{ }^{13} \mathrm{C} \mathrm{NMR}\left(50 \mathrm{MHz}, \mathrm{CDCl}_{3}\right)$ : $\delta 199.8,141.5,138.6,130.3,128.2,127.5,127.4,109.2,80.6,80.5,72.8,70.4,40.9,32.1,31.9,29.7$, 29.6, 29.5, 29.3, 29.2, 27.3, 26.7, 26.2, 26.0, 23.9, 22.6, 14.1; MS (ESI) m/z: Calcd for $\left(\mathrm{M}^{+}+\mathrm{Na}\right)$ 607.48. Found: 607.03; Anal. Calcd for $\mathrm{C}_{38} \mathrm{H}_{64} \mathrm{O}_{4}$ : C, 78.03; H, 11.03. Found: C, 77.93; H, 11.09. 


\section{(S,E)-16-(Benzyloxy)-1-((4S,5S)-5-decyl-2,2-dimethyl-1,3-dioxolan-4-yl)hexadec-1-en-3-ol (24).}

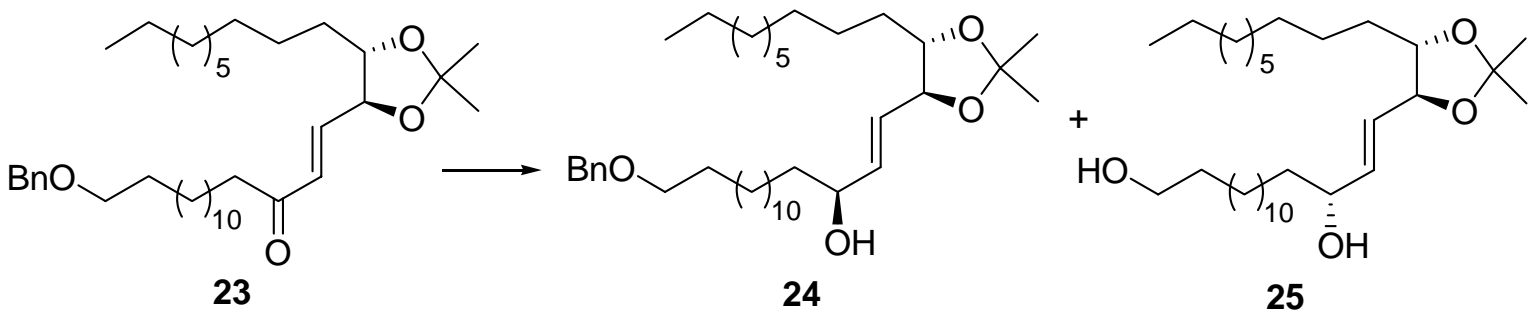

The (S)-( -)-BINAL-H reagent was prepared by stirring LAH (1.6 M THF solution, $3.2 \mathrm{~mL}, 5.1 \mathrm{mmol})$, ethanol (2.0 M THF solution, $2.55 \mathrm{~mL}, 5.1 \mathrm{mmol})$ and (-)-binapthol (1.46 g, $5.1 \mathrm{mmol})$ in THF (15 mL) for $30 \mathrm{~min}$ at room temperature. It was then cooled to $-78{ }^{\circ} \mathrm{C}, 23(1.0 \mathrm{~g}, 1.7 \mathrm{mmol})$ in $\mathrm{THF}(3 \mathrm{~mL})$ was added drop wise over a period of $15 \mathrm{~min}$. After $2.5 \mathrm{~h}$, methanol $(2 \mathrm{~mL})$ was introduced and the reaction mixture was allowed to attain room temperature. After the addition of $2 \mathrm{~N} \mathrm{HCl}(1.5 \mathrm{~mL})$, it was extracted with ether $(2 \times 15 \mathrm{~mL})$. The ether layer was washed with sodium bicarbonate solution, water, dried over anhydrous $\mathrm{Na}_{2} \mathrm{SO}_{4}$ and evaporated. The residue was purified on silica gel by eluting with ethyl acetate and light petroleum (1:9) to afford $24(770 \mathrm{mg}, 77 \%)$ as colorless oil. $[\alpha]_{\mathrm{D}}=-0.8(c 1.5$, $\left.\mathrm{CHCl}_{3}\right)$; IR $\left(\mathrm{CHCl}_{3}\right) v_{\max }\left(\mathrm{cm}^{-1}\right) 3446,3012,2988,2855,1647,1495,1380 ;{ }^{1} \mathrm{H} \mathrm{NMR}(400 \mathrm{MHz}$, $\left.\mathrm{CDCl}_{3}\right): \delta 7.33-7.26(\mathrm{~m}, 5 \mathrm{H}), 5.83(\mathrm{dd}, J=15.6,5.7 \mathrm{~Hz}, 1 \mathrm{H}), 5.63(\mathrm{dd}, J=15.6,7.3 \mathrm{~Hz}, 1 \mathrm{H}), 4.49(\mathrm{~s}$, 2H), $4.13(\mathrm{q}, J=6.0 \mathrm{~Hz}, 1 \mathrm{H}), 3.97(\mathrm{t}, J=7.8 \mathrm{~Hz}, 1 \mathrm{H}), 3.65(\mathrm{ddd}, J=8.3,6.0,5.7 \mathrm{~Hz}, 1 \mathrm{H}), 3.45(\mathrm{t}, J=$ $6.6 \mathrm{~Hz}, 2 \mathrm{H}), 1.64-1.59(\mathrm{~m}, 2 \mathrm{H}), 1.54-1.49(\mathrm{~m}, 4 \mathrm{H}), 1.40(\mathrm{~s}, 3 \mathrm{H}), 1.39(\mathrm{~s}, 3 \mathrm{H}), 1.26(\mathrm{~m}, 36 \mathrm{H}), 0.88(\mathrm{t}, J$ $=6.7 \mathrm{~Hz}, 3 \mathrm{H}) ;{ }^{13} \mathrm{C} \mathrm{NMR}\left(100 \mathrm{MHz}, \mathrm{CDCl}_{3}\right): \delta 138.7,137.9,128.3,127.6,127.4,127.3,108.4,81.9$, 80.9, 72.8, 71.8, 70.5, 37.2, 32.0, 29.8 (2C), 29.7, 29.6 (2C), 29.5 (2C), 29.4, 27.3, 27.0, 26.2, 26.1, 25.3, 22.7, 14.1; MS (ESI) m/z: Calcd for $\left(\mathrm{M}^{+}+\mathrm{Na}\right)$ 609.5. Found: 609.47; Anal. Calcd for $\mathrm{C}_{38} \mathrm{H}_{66} \mathrm{O}_{4}$ : C, 77.76; H, 11.33. Found: C, 77.52; H, 11.10.

Further elution gave (R,E)-16-(Benzyloxy)-1-((4S,5S)-5-decyl-2,2-dimethyl-1,3-dioxolan-4-yl)hexadec-1-en-3-ol (25). (70 mg, 7\%), $[\alpha]_{\mathrm{D}}=-7.7\left(c \mathrm{1.3}, \mathrm{CHCl}_{3}\right)$; IR $\left(\mathrm{CHCl}_{3}\right) v_{\max }\left(\mathrm{cm}^{-1}\right) 3446,3012,2988$, 
2855, 1647, 1495, 1380; ${ }^{1} \mathrm{H}$ NMR (200 MHz, $\left.\mathrm{CDCl}_{3}\right): \delta 7.34-7.26(\mathrm{~m}, 5 \mathrm{H}), 5.81(\mathrm{dd}, J=15.3,6.0 \mathrm{~Hz}$, 1H), $5.61(\mathrm{dd}, J=15.3,7.2 \mathrm{~Hz}, 1 \mathrm{H}), 4.49(\mathrm{~s}, 2 \mathrm{H}), 4.14-4.06(\mathrm{~m}, 1 \mathrm{H}), 4.01-4.93(\mathrm{~m}, 1 \mathrm{H}), 3.68-3.59(\mathrm{~m}$, 1H), $3.45(\mathrm{t}, J=6.6 \mathrm{~Hz}, 2 \mathrm{H}), 1.67-1.50(\mathrm{~m}, 8 \mathrm{H}), 1.40(\mathrm{~s}, 6 \mathrm{H}), 1.26(\mathrm{~m}, 34 \mathrm{H}), 0.88(\mathrm{t}, J=6.7 \mathrm{~Hz}, 3 \mathrm{H})$; ${ }^{13} \mathrm{C}$ NMR $\left(50 \mathrm{MHz}, \mathrm{CDCl}_{3}\right): \delta 138.7,138.0,128.3,127.5,127.4,108.4,81.9,80.7,72.8,72.1,70.4$, 37.1, 31.9, 29.7, 29.6, 29.5, 29.4, 27.3, 27.0, 26.2, 25.4, 22.7, 14.1; MS (ESI) m/z: Calcd for $\left(\mathrm{M}^{+}+\mathrm{Na}\right)$ 609.5. Found: 609.47. Anal. Calcd for $\mathrm{C}_{38} \mathrm{H}_{66} \mathrm{O}_{4}$ : C, 77.76; H, 11.33. Found: C, 77.50; H, 11.33.

\section{(S)-16-((4S,5S)-5-Decyl-2,2-dimethyl-1,3-dioxolan-4-yl)-14-(methoxymethoxy)hexadec-an-1-ol}

(26).

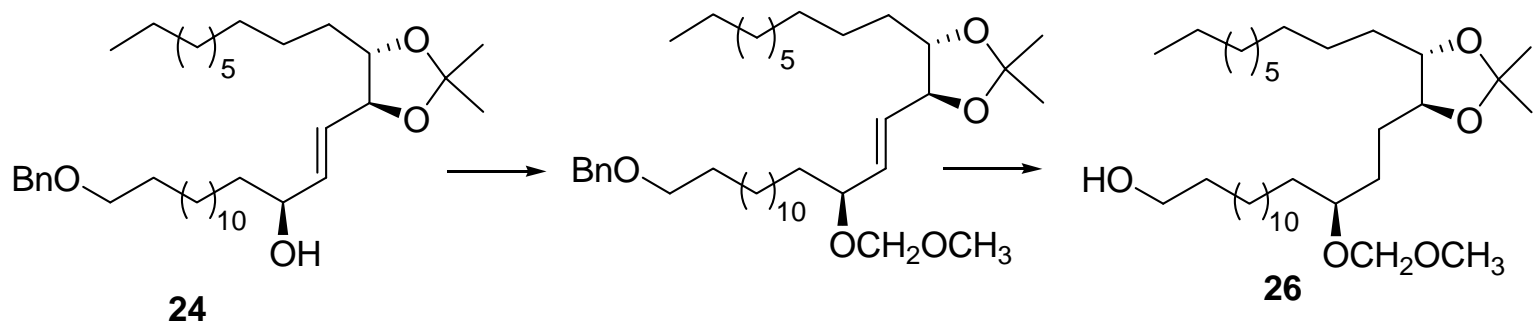

Compound 24 (500 mg, $0.8 \mathrm{mmol})$, MOM-chloride $(0.2 \mathrm{~mL}, 1.8 \mathrm{mmol})$ and Hunig's base $(0.4 \mathrm{~mL}, 2.2$ mmol) in $\mathrm{CH}_{2} \mathrm{Cl}_{2}(4 \mathrm{~mL})$ were stirred at room temperature for $2 \mathrm{~h}$, washed with water, dried over anhydrous $\mathrm{Na}_{2} \mathrm{SO}_{4}$ and concentrated. The residue was purified on silica gel (pre treated with $\mathrm{Et}_{3} \mathrm{~N}$ ) using ethyl acetate and light petroleum (0.5:9.5) to furnish the MOM-derivative (520 mg, 97\%) as yellow oil. $[\alpha]_{\mathrm{D}}=-49.8\left(\right.$ ( $\left.1.0, \mathrm{CHCl}_{3}\right) ; \mathrm{IR}\left(\mathrm{CHCl}_{3}\right) v_{\max }\left(\mathrm{cm}^{-1}\right) 2928,2855,1601,1495,1465,1379 ;{ }^{1} \mathrm{H}$ NMR (400 MHz, $\left.\mathrm{CDCl}_{3}\right): \delta 7.33-7.26(\mathrm{~m}, 5 \mathrm{H}), 5.62-5.60(\mathrm{~m}, 2 \mathrm{H}), 4.64(\mathrm{~d}, J=6.8 \mathrm{~Hz}, 1 \mathrm{H}), 4.51(\mathrm{~d}, J$ $=6.8 \mathrm{~Hz}, 1 \mathrm{H}), 4.49(\mathrm{~s}, 2 \mathrm{H}), 4.04-3.96(\mathrm{~m}, 2 \mathrm{H}), 3.67-3.62(\mathrm{~m}, 1 \mathrm{H}), 3.45(\mathrm{t}, J=6.6 \mathrm{~Hz}, 2 \mathrm{H}), 3.35(\mathrm{~s}$, $3 \mathrm{H}), 1.64-1.50(\mathrm{~m}, 6 \mathrm{H}), 1.40(\mathrm{~s}, 3 \mathrm{H}), 1.39(\mathrm{~s}, 3 \mathrm{H}), 1.26(\mathrm{~m}, 36 \mathrm{H}), 0.88(\mathrm{t}, J=6.7 \mathrm{~Hz}, 3 \mathrm{H}) ;{ }^{13} \mathrm{C} \mathrm{NMR}$ $\left(100 \mathrm{MHz}_{\mathrm{CDCl}}\right): \delta 138.7,134.9,130.1,128.3,127.6,127.4,108.4,93.7,81.9,80.8,75.9,72.9,70.5$, 55.4, 35.6, 31.9, 29.8 (2C), 29.5, 29.4, 27.3, 27.0, 26.2, 26.1, 25.4, 22.7, 14.2; MS (ESI) m/z: Calcd for $\left(\mathrm{M}^{+}+\mathrm{Na}\right)$ 653.52. Found: 652.87. Anal. Calcd for $\mathrm{C}_{40} \mathrm{H}_{70} \mathrm{O}_{5}$ : C, 76.14; $\mathrm{H}, 11.18$. Found: $\mathrm{C}, 75.96 ; \mathrm{H}$, 11.10 . 
The MOM-derivative $(400 \mathrm{mg}, 0.6 \mathrm{mmol}), 10 \% \mathrm{Pd}(\mathrm{OH})_{2} / \mathrm{C}(50 \mathrm{mg})$ in ethyl acetate $(4 \mathrm{~mL})$ were hydrogenated at normal temperature and pressure. After $6 \mathrm{~h}$, the reaction mixture was filtered through a pad of Celite and concentrated. The residue was purified on silica gel using ethyl acetate and light petroleum (1:4) to provide $\mathbf{2 6}(270 \mathrm{mg}, 80 \%)$ as a colorless oil:

$[\alpha]_{\mathrm{D}}=-13.3\left(c\right.$ 1.9, $\left.\mathrm{CHCl}_{3}\right) ; \mathrm{IR}\left(\mathrm{CHCl}_{3}\right) v_{\max }\left(\mathrm{cm}^{-1}\right) 3425,2927,2854,1613,1465,1378 ;{ }^{1} \mathrm{H} \mathrm{NMR}(500$ $\left.\mathrm{MHz}, \mathrm{CDCl}_{3}\right): \delta 4.64(\mathrm{~s}, 2 \mathrm{H}), 3.62(\mathrm{t}, J=6.9 \mathrm{~Hz}, 2 \mathrm{H}), 3.58-3.52(\mathrm{~m}, 3 \mathrm{H}), 3.37(\mathrm{~s}, 3 \mathrm{H}), 1.73-1.44(\mathrm{~m}$, 14H), $1.36(\mathrm{~s}, 6 \mathrm{H}), 1.26(\mathrm{~m}, 32 \mathrm{H}), 0.88(\mathrm{t}, J=6.7 \mathrm{~Hz}, 3 \mathrm{H}) ;{ }^{13} \mathrm{C} \mathrm{NMR}\left(125 \mathrm{MHz}, \mathrm{CDCl}_{3}\right): \delta 107.8,95.5$, 81.2, 81.0, 77.5, 62.9, 55.5, 34.5, 33.0, 32.8, 31.9, 31.0, 29.8 (2C), 29.6, 29.4, 29.3, 28.8, 27.3, 26.1, 25.8, 25.2, 22.7, 14.1; MS (ESI) m/z: Calcd for $\left(\mathrm{M}^{+}+\mathrm{Na}\right)$ 565.49. Found: 565.73; Anal. Calcd for $\mathrm{C}_{33} \mathrm{H}_{66} \mathrm{O}_{5}:$ C, 73.01; H, 12.25. Found: C, 73.15; H, 12.12.

\section{(S)-N-((3S,11bS)-9,11-bis(benzyloxy)-4-oxo-2,3,4,6,7,11b-hexahydro-1H-pyrido[2,1-a]isoquinolin-}

\section{3-yl)-16-((4S,5S)-5-decyl-2,2-dimethyl-1,3-dioxolan-4-yl)-14-(methoxymethoxy)hexadecanamide} (27).

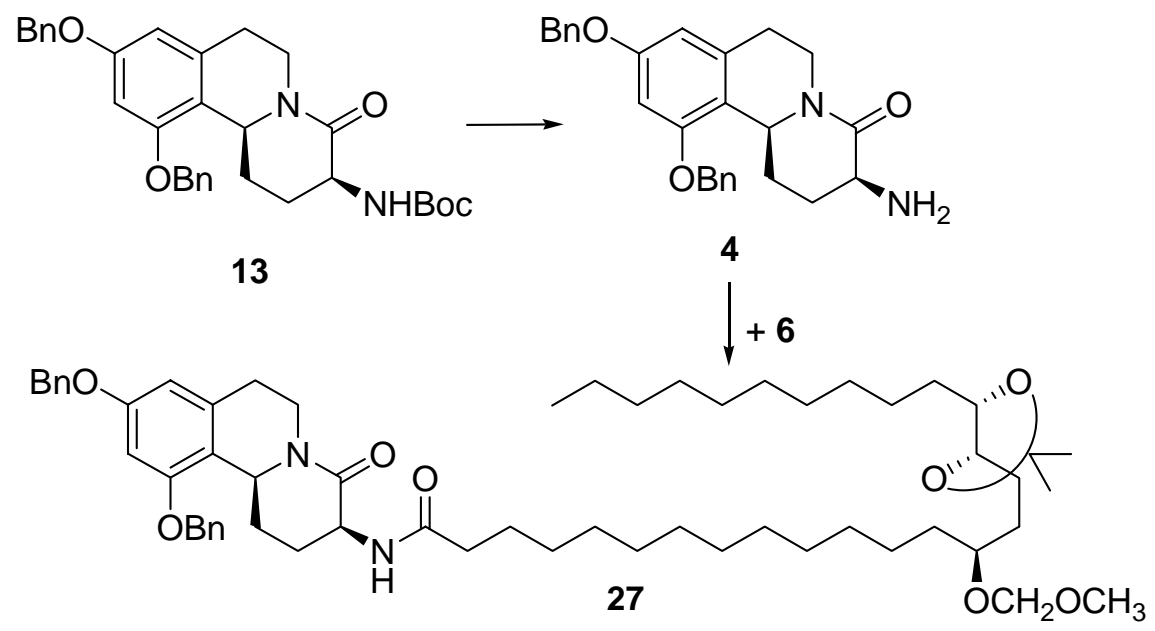

A suspension of compound $13(450 \mathrm{mg}, 0.84 \mathrm{mmol})$ and $3 \mathrm{~N}-\mathrm{HCl}$ in ethyl acetate $(8 \mathrm{~mL})$ were stirred for $3 \mathrm{~h}$ at room temperature, diluted with ethyl acetate and neutralized with saturated sodium bicarbonate solution. The aqueous phase was extracted with ethyl acetate $(10 \mathrm{~mL}$ x 3). The combined organic layer was dried over anhydrous $\mathrm{Na}_{2} \mathrm{SO}_{4}$ and concentrated to give the free amine 4 (340 mg). 
To a solution of 6 (200 mg, $0.36 \mathrm{mmol})$, HOBt $(73 \mathrm{mg}, 0.54 \mathrm{mmol})$, EDC (90 mg, $0.47 \mathrm{mmol})$ in dry dichloromethane $(5 \mathrm{~mL})$ was added a solution of $4(170 \mathrm{mg}, 0.39 \mathrm{mmol})$ in dry dichloromethane $(2 \mathrm{~mL})$ followed by dry triethylamine $(0.1 \mathrm{~mL})$. After stirring for $15 \mathrm{~h}$ at room temperature, the reaction mixture was diluted with water, extracted with dichloromethane $(10 \mathrm{~mL} \times 2)$. The combined organic layer was washed with water, dried over anhydrous $\mathrm{Na}_{2} \mathrm{SO}_{4}$ and concentrated to give a crude oil which was purified on silica gel using ethyl acetate and light petroleum (1:3) to afford $27(252 \mathrm{mg}, 72 \%)$ as a color less liquid. $[\alpha]_{\mathrm{D}}=-52.9\left(c\right.$ 0.65, $\left.\mathrm{CHCl}_{3}\right) ; \mathrm{IR}\left(\mathrm{CHCl}_{3}\right) v_{\max }\left(\mathrm{cm}^{-1}\right) 3395,3011,2926,2854,1731,1646$, 1609, 1498, 1465, 1378, 1150, 1095, 1040; ${ }^{1} \mathrm{H}$ NMR (400 MHz, $\left.\mathrm{CDCl}_{3}\right): \delta 7.41-7.32(\mathrm{~m}, 10 \mathrm{H}), 6.78$ $(\mathrm{d}, J=5.3 \mathrm{~Hz}, 1 \mathrm{H}), 6.46(\mathrm{~d}, J=2.2 \mathrm{~Hz}, 1 \mathrm{H}), 6.39(\mathrm{~d}, J=2.2 \mathrm{~Hz}, 1 \mathrm{H}), 5.09(\mathrm{~s}, 2 \mathrm{H}), 4.99(\mathrm{~s}, 2 \mathrm{H}), 4.93$ $(\mathrm{dd}, J=10.5,4.0 \mathrm{~Hz}, 1 \mathrm{H}), 4.79-4.70(\mathrm{~m}, 1 \mathrm{H}), 4.65(\mathrm{~s}, 2 \mathrm{H}), 4.54(\mathrm{~m}, 1 \mathrm{H}), 3.65-3.49(\mathrm{~m}, 3 \mathrm{H}), 3.38(\mathrm{~s}$, 3H), 2.89-2.62 (m, 4H), 2.57-2.43 (m, 1H), $2.25(\mathrm{t}, J=7.5 \mathrm{~Hz}, 2 \mathrm{H}), 1.79-1.45(\mathrm{~m}, 15 \mathrm{H}), 1.37(\mathrm{~s}, 6 \mathrm{H})$, $1.35-1.20(\mathrm{~m}, 31 \mathrm{H}), 0.88(\mathrm{t}, J=6.5 \mathrm{~Hz}, 3 \mathrm{H}) ;{ }^{13} \mathrm{C}\left(50 \mathrm{MHz}, \mathrm{CDCl}_{3}\right): \delta 172.9,170.4,158.3,155.8$, $137.0,136.5,136.3,128.7,128.5,128.0,127.9,127.3,126.9,117.0,107.6,105.7,98.9,95.3,81.1,80.9$, $77.4,70.0,55.4,48.6,38.7,36.6,34.3,32.8,31.8,30.8,29.7,29.6,29.5,29.4,29.3,29.2,29.2,29.1$, 28.7, 28.4, 27.2, 26.0, 25.6, 25.1, 25.1, 22.5, 14.0; MS (ESI) m/z: Calcd for $\left(\mathrm{M}^{+}+\mathrm{Na}\right)$ 989.67. Found: 989.75; Anal. Calcd for $\mathrm{C}_{60} \mathrm{H}_{90} \mathrm{~N}_{2} \mathrm{O}_{8}$ : C, 74.50; H, 9.38; N, 2.90. Found: C, 74.33; H, 9.25; N, 2.84.

(14S,17S,18S)-N-((3S,11bS)-9,11-Bis(benzyloxy)-4-oxo-2,3,4,6,7,11b-hexahydro-1H-pyrido[2,1a]isoquinolin-3-yl)-14,17,18-trihydroxyoctacosanamide (28).

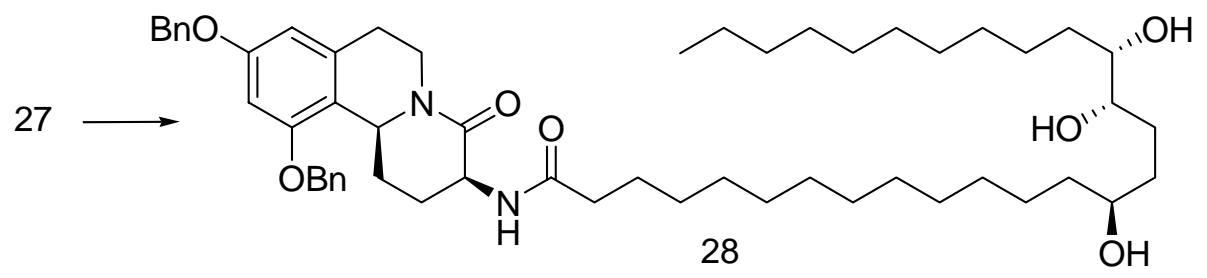

A solution of 27 (120 mg, $0.12 \mathrm{mmol})$ and TMSI (freshly prepared from TMS-Cl and sodium iodide in acetonitrile $)(0.5 \mathrm{~g}, 0.25 \mathrm{mmol})$ in dichloromethane $(6 \mathrm{~mL})$ and acetonitrile $(2 \mathrm{~mL})$ under nitrogen at 0 
${ }^{\circ} \mathrm{C}$ was stirred for $1 \mathrm{~h}$ and then $10 \% \mathrm{NaHCO}_{3}$ solution was added and layers separated. The aqueous layer was extracted with dichloromethane $(25 \mathrm{~mL}$ x 3), the combined organic layer was dried over anhydrous $\mathrm{Na}_{2} \mathrm{SO}_{4}$, concentrated and purified on silica gel by using ethyl acetate to afford 28 (44 mg, 47\%). $[\alpha]_{\mathrm{D}}=-79.4\left(c\right.$ 0.5, $\left.\mathrm{CHCl}_{3}\right)$; IR $\left(\mathrm{CHCl}_{3}\right) v_{\max }\left(\mathrm{cm}^{-1}\right) 3394,3016,2925,2853,1636,1608,1498$, 1465, 1375, 1358, 1308, 1271, 1151, 1090, 1048; ${ }^{1} \mathrm{H}$ NMR (400 MHz, $\left.\mathrm{CDCl}_{3}\right): \delta 7.41-7.33(\mathrm{~m}, 10 \mathrm{H})$, $6.78(\mathrm{~d}, J=5.4 \mathrm{~Hz}, 1 \mathrm{H}), 6.47(\mathrm{~d}, J=2.2 \mathrm{~Hz}, 1 \mathrm{H}), 6.39(\mathrm{~d}, J=2.2 \mathrm{~Hz}, 1 \mathrm{H}), 5.09(\mathrm{~s}, 1 \mathrm{H}), 5.09(\mathrm{~s}, 1 \mathrm{H})$, $4.99(\mathrm{~s}, 2 \mathrm{H}), 4.94(\mathrm{dd}, J=10.7,4.2 \mathrm{~Hz}, 1 \mathrm{H}), 4.74-4.70(\mathrm{~m}, 1 \mathrm{H}), 4.53(\mathrm{~m}, 1 \mathrm{H}), 3.70-3.64(\mathrm{~m}, 1 \mathrm{H}), 3.47-$ $3.42(\mathrm{~m}, 2 \mathrm{H}), 2.86-2.75(\mathrm{~m}, 2 \mathrm{H}), 2.72-2.65(\mathrm{~m}, 2 \mathrm{H}), 2.52-2.47(\mathrm{~m}, 1 \mathrm{H}), 2.25(\mathrm{t}, J=7.6,2 \mathrm{H}), 1.72-1.40$ $(\mathrm{m}, 15 \mathrm{H}), 1.37-1.25(\mathrm{~m}, 31 \mathrm{H}), 0.88(\mathrm{t}, J=6.8 \mathrm{~Hz}, 3 \mathrm{H}) ;{ }^{13} \mathrm{C}\left(100 \mathrm{MHz}, \mathrm{CDCl}_{3}\right): \delta 173.1,170.5,158.5$, $155.9,137.1,136.7,136.4,128.8,128.6,128.1,128.1,127.5,127.0,117.2,105.8,99.1,74.6,74.4,71.9$, $70.1,48.8,38.9,37.5,36.7,33.5,33.0,31.8,29.6,29.5,29.5,29.4,29.4,29.3,29.2,28.5,25.7,25.6$, 25.2, 22.6, 14.0; MS (ESI) m/z: Calcd for $\left(\mathrm{M}^{+}+\mathrm{Na}\right)$ 905.61. Found: 905.38; Anal. Calcd for $\mathrm{C}_{55} \mathrm{H}_{82} \mathrm{~N}_{2} \mathrm{O}_{7}: \mathrm{C}, 74.79 ; \mathrm{H}, 9.36 ; \mathrm{N}, 3.17$. Found: C, 74.82; H, 9.18; N, 2.92.

(S)-N-((3S,11bR)-9,11-Bis(benzyloxy)-4-oxo-2,3,4,6,7,11b-hexahydro-1H-pyrido[2,1-a]isoquinolin3-yl)-16-((4S,5S)-5-decyl-2,2-dimethyl-1,3-dioxolan-4-yl)-14(methoxymethoxy)hexadec-anamide (29).

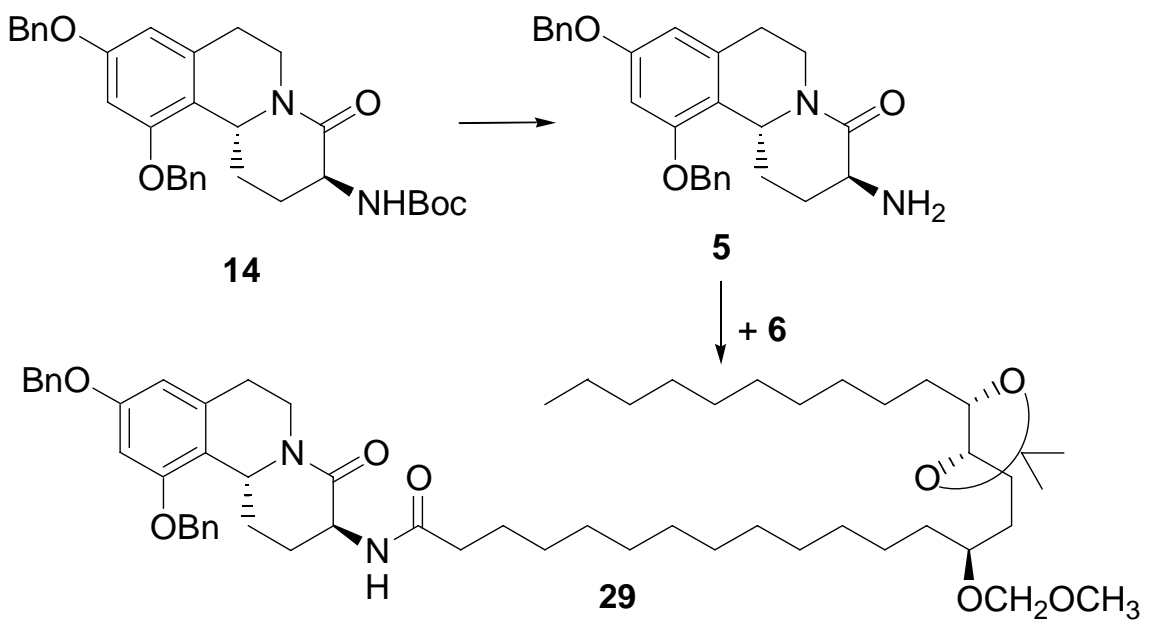


The free amine (5) was prepared as described above from compound $\mathbf{1 4}$ and used immediately for the next reaction.

Compound 29 was prepared by applying the same procedure as described for 27.

$[\alpha]_{\mathrm{D}}=+76.7\left(c\right.$ 2.4, $\left.\mathrm{CHCl}_{3}\right)$; IR $\left(\mathrm{CHCl}_{3}\right) v_{\max }\left(\mathrm{cm}^{-1}\right) 3317,3009,2928,2855,1635,1607,1498,1463$, 1378, 1307, 1269, 1151, 1093, 1039; ${ }^{1} \mathrm{H}$ NMR $\left(400 \mathrm{MHz}, \mathrm{CDCl}_{3}\right): \delta 7.39-7.31(\mathrm{~m}, 10 \mathrm{H}), 6.48(\mathrm{~d}, J=$ $2.1 \mathrm{~Hz}, 1 \mathrm{H}), 6.35(\mathrm{~d}, J=2.1 \mathrm{~Hz}, 1 \mathrm{H}), 6.30(\mathrm{~d}, J=4.9 \mathrm{~Hz}, 1 \mathrm{H}), 5.07-4.97(\mathrm{~m}, 4 \mathrm{H}), 4.93-4.88(\mathrm{~m}, 1 \mathrm{H})$, $4.78(\mathrm{dd}, J=10.9,3.6 \mathrm{~Hz}, 1 \mathrm{H}), 4.64(\mathrm{~s}, 2 \mathrm{H}), 4.19(\mathrm{~m}, 1 \mathrm{H}), 3.63-3.48(\mathrm{~m}, 3 \mathrm{H}), 3.37(\mathrm{~s}, 3 \mathrm{H}), 3.07(\mathrm{dq}, J=$ 13.7, $3.5 \mathrm{~Hz}, 1 \mathrm{H}), 2.91-2.77(\mathrm{~m}, 1 \mathrm{H}), 2.67-2.54(\mathrm{~m}, 3 \mathrm{H}), 2.21(\mathrm{t}, J=7.5 \mathrm{~Hz}, 2 \mathrm{H}), 1.77-1.59(\mathrm{~m}, 7 \mathrm{H})$, $1.55-1.42(\mathrm{~m}, 8 \mathrm{H}), 1.36(\mathrm{~s}, 6 \mathrm{H}), 1.34-1.20(\mathrm{~m}, 31 \mathrm{H}), 0.88(\mathrm{t}, J=6.7 \mathrm{~Hz}, 3 \mathrm{H}) ;{ }^{13} \mathrm{C}\left(50 \mathrm{MHz}, \mathrm{CDCl}_{3}\right): \delta$ $173.5,168.7,158.1,156.7,137.7,136.6,136.3,128.6,128.5,128.5,128.0,127.9,127.3,127.0,118.1$, 107.7, 105.9, 99.1, 95.4, 81.1, 80.9, 77.4, 70.1, 69.9, 56.1, 55.4, 51.9, 39.5, 36.6, 34.4, 32.9, 31.8, 30.9, $30.5,29.8,29.7,29.6,29.5,29.5,29.4,29.3,29.3,29.2,28.8,27.9,27.3,26.1,25.6,25.2,22.6,14.1$; MS (ESI) m/z: Calcd for $\left(\mathrm{M}^{+}+\mathrm{Na}\right)$ 989.67. Found: 989.75; Anal. Calcd for $\mathrm{C}_{60} \mathrm{H}_{90} \mathrm{~N}_{2} \mathrm{O}_{8}: \mathrm{C}, 74.50 ; \mathrm{H}$, 9.38; N, 2.90. Found: C, 74.68; H, 9.17; N, 2.87.

\section{(14S,17S,18S)-N-((3S,11bR)-9,11-Bis(benzyloxy)-4-oxo-2,3,4,6,7,11b-hexahydro-1H-pyrido[2,1-} a]isoquinolin-3-yl)-14,17,18-trihydroxyoctacosanamide (30).

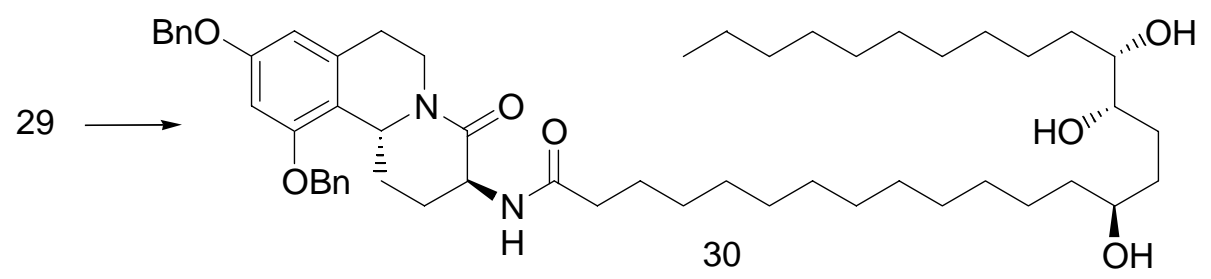

Compound $\mathbf{3 0}$ was prepared by applying the same procedure as described for $\mathbf{2 8}$.

$[\alpha]_{\mathrm{D}}=+92.3\left(c 1.2, \mathrm{CHCl}_{3}\right) ; \mathrm{IR}\left(\mathrm{CHCl}_{3}\right) v_{\max }\left(\mathrm{cm}^{-1}\right) 3394,3016,2925,2853,1636,1608,1498,1465$, 1375, 1358, 1308, 1271, 1151, 1090, 1048; ${ }^{1} \mathrm{H}$ NMR (400 MHz, $\left.\mathrm{CDCl}_{3}\right): \delta 7.43-7.32(\mathrm{~m}, 10 \mathrm{H}), 6.50(\mathrm{~d}$, $J=2.2 \mathrm{~Hz}, 1 \mathrm{H}), 6.37(\mathrm{~d}, J=2.2 \mathrm{~Hz}, 1 \mathrm{H}), 5.08-5.00(\mathrm{~m}, 4 \mathrm{H}), 4.94-4.88(\mathrm{~m}, 1 \mathrm{H}), 4.79$ (dd, $J=11.0,3.2$ 
Hz, 1H), $4.21(\mathrm{~m}, 1 \mathrm{H}), 3.65(\mathrm{~m}, 1 \mathrm{H}), 3.44(\mathrm{~m}, 2 \mathrm{H}), 3.11-3.04(\mathrm{~m}, 1 \mathrm{H}), 2.91-2.78(\mathrm{~m}, 1 \mathrm{H}), 2.68-2.52$ $(\mathrm{m}, 3 \mathrm{H}), 2.22(\mathrm{t}, J=7.6 \mathrm{~Hz}, 2 \mathrm{H}), 1.71-1.60(\mathrm{~m}, 7 \mathrm{H}), 1.57-1.38(\mathrm{~m}, 8 \mathrm{H}), 1.36-1.20(\mathrm{~m}, 31 \mathrm{H}), 0.88(\mathrm{t}, J$ $=6.8 \mathrm{~Hz}, 3 \mathrm{H}) ;{ }^{13} \mathrm{C}\left(100 \mathrm{MHz}, \mathrm{CDCl}_{3}\right): \delta 173.7,168.8,158.2,156.8,137.7,136.6,136.4,128.6,128.6$, $128.5,128.1,127.5,127.1,118.1,106.1,99.1,74.5,74.4,71.8,70.2,70.1,56.2,51.9,39.5,37.4,36.7$, $33.5,33.1,31.9,30.6,29.7,29.6,29.5,29.3,29.2,29.2,27.7,27.2,25.7,25.6,25.6,22.7,14.1$; MS (ESI) m/z: Calcd for $\left(\mathrm{M}^{+}+\mathrm{Na}\right)$ 905.61. Found: 905.38; Anal. Calcd for $\mathrm{C}_{55} \mathrm{H}_{82} \mathrm{~N}_{2} \mathrm{O}_{7}$ : C, 74.79; $\mathrm{H}, 9.36$; N, 3.17. Found: C, 74.65; H, 9.22; N, 3.12.

\section{Synthesis of Schulzeine C (3)}

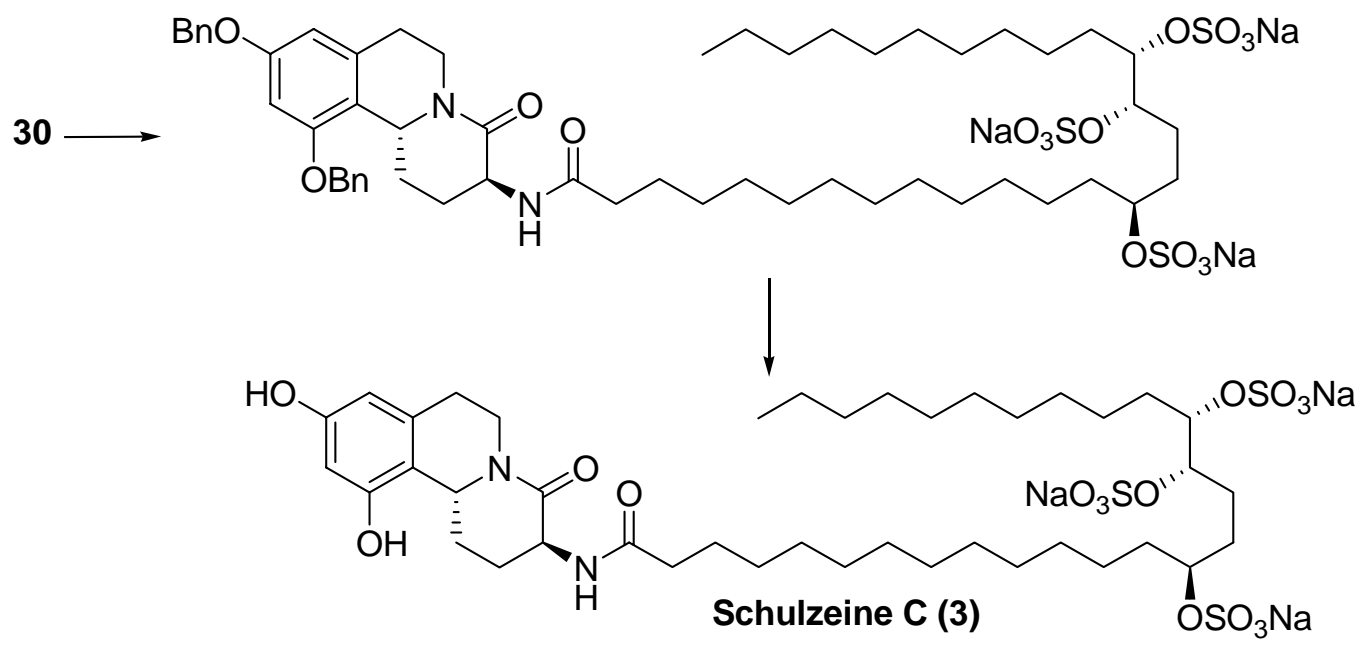

Compound $\mathbf{3}$ was prepared from $\mathbf{3 0}$ by applying the procedure described for 2.

For sulfate salt, $[\alpha]_{\mathrm{D}}=+24.1\left(c 1, \mathrm{CH}_{3} \mathrm{OH}\right) ; \mathrm{IR}\left(\mathrm{CH}_{3} \mathrm{OH}\right) v_{\max }\left(\mathrm{cm}^{-1}\right) 2947,2835,1650,1449,1418$, 1220, 1113, 1026; ${ }^{1} \mathrm{H}$ NMR (400 MHz, $\left.\mathrm{CD}_{3} \mathrm{OD}\right): \delta 7.42-7.29(\mathrm{~m}, 10 \mathrm{H}), 6.58(\mathrm{~d}, J=2.2 \mathrm{~Hz}, 1 \mathrm{H}), 6.42$ $(\mathrm{d}, J=2.2 \mathrm{~Hz}, 1 \mathrm{H}), 5.12-5.04(\mathrm{~m}, 4 \mathrm{H}), 4.77(\mathrm{~m}, 2 \mathrm{H}), 4.67(\mathrm{~m}, 2 \mathrm{H}), 4.35(\mathrm{dt}, J=11.1,5.7 \mathrm{~Hz}, 1 \mathrm{H}), 4.22$ $(\mathrm{dd}, J=11.5,6.9 \mathrm{~Hz}, 1 \mathrm{H}), 2.95(\mathrm{dq}, J=13.5,3.2 \mathrm{~Hz}, 1 \mathrm{H}), 2.77(\mathrm{~m}, 1 \mathrm{H}), 2.66-2.60(\mathrm{~m}, 2 \mathrm{H}), 2.22(\mathrm{dt}, J$ $=7.4,2.2 \mathrm{~Hz}, 2 \mathrm{H}), 2.06(\mathrm{~m}, 1 \mathrm{H}), 1.94(\mathrm{~m}, 1 \mathrm{H}), 1.90(\mathrm{~m}, 1 \mathrm{H}), 1.75-1.51(\mathrm{~m}, 10 \mathrm{H}), 1.40(\mathrm{~m}, 4 \mathrm{H}), 1.27$ (m, 30H), $0.88(\mathrm{t}, J=6.7 \mathrm{~Hz}, 3 \mathrm{H}) ;{ }^{13} \mathrm{C}\left(100 \mathrm{MHz}, \mathrm{CD}_{3} \mathrm{OD}\right): \delta 176.1,170.5,159.7,158.0,139.0,138.6$, 
$138.3,129.6,129.5,129.0,128.8,128.5,128.4,119.1,107.7,100.2,81.2,80.0,79.9,71.3,71.1,56.9$, $52.0,40.6,37.1,35.54,31.7,31.2,30.8,30.8,30.7,30.7,30.7,30.6,30.4,30.4,30.2,29.9,29.8,28.7$, 28.4, 26.9, 26.8, 25.9, 25.8, 23.7, 14.4; MS (ESI) m/z: Calcd for $\left(\mathrm{M}^{+}+\mathrm{Na}\right)$ 1211.43. Found: 1211.48.

For Schulzeine C (2), $[\alpha]_{\mathrm{D}}=+35.5\left(c 1.2, \mathrm{CH}_{3} \mathrm{OH}\right)$, Lit $[\alpha]_{\mathrm{D}}=+33\left(c 0.1, \mathrm{CH}_{3} \mathrm{OH}\right)$; IR (nujol) $v_{\max }\left(\mathrm{cm}^{-}\right.$ $\left.{ }^{1}\right) 3341,2923,2853,1603,1462,1376,1253,1220,1150,1063,951 ;{ }^{1} \mathrm{H}$ NMR (400 MHz, $\left.\mathrm{CD}_{3} \mathrm{OD}\right): \delta$ $6.19(\mathrm{~d}, J=2.3 \mathrm{~Hz}, 1 \mathrm{H}), 6.09(\mathrm{~d}, J=2.3 \mathrm{~Hz}, 1 \mathrm{H}), 4.79(\mathrm{~m}, 2 \mathrm{H}), 4.66(\mathrm{~m}, 2 \mathrm{H}), 4.35(\mathrm{q}, J=5.6 \mathrm{~Hz}, 1 \mathrm{H})$, $4.28(\mathrm{dd}, J=11.8,6.8 \mathrm{~Hz}, 1 \mathrm{H}), 3.07(\mathrm{dq}, J=14.0,3.4 \mathrm{~Hz}, 1 \mathrm{H}), 2.69$ (d, $J=13.2 \mathrm{~Hz}, 1 \mathrm{H}), 2.63$ (dt, $J=$ 11.7, $2.0 \mathrm{~Hz}, 1 \mathrm{H}), 2.53(\mathrm{~d}, J=16.6 \mathrm{~Hz}, 1 \mathrm{H}), 2.23(\mathrm{dt}, J=7.4,2.4 \mathrm{~Hz}, 2 \mathrm{H}), 2.09(\mathrm{~m}, 1 \mathrm{H}), 1.94(\mathrm{~m}, 2 \mathrm{H})$, $1.75-1.50(\mathrm{~m}, 10 \mathrm{H}), 1.42-1.39(\mathrm{~m}, 4 \mathrm{H}), 1.36-1.25(\mathrm{~m}, 30 \mathrm{H}), 0.88(\mathrm{t}, J=6.8 \mathrm{~Hz}, 3 \mathrm{H}) ;{ }^{13} \mathrm{C}(100 \mathrm{MHz}$, $\left.\mathrm{CD}_{3} \mathrm{OD}\right): \delta 176.1,170.5,157.6,156.7,138.6,115.9,107.6,102.1,81.2,80.0,79.9,57.2,52.0,40.8$, $37.1,35.4,31.7,30.8,30.8,30.7,30.7,30.7,30.6,30.4,30.4,30.2,29.8,29.4,28.4,26.9,26.8,25.9$, 25.8, 23.7, 14.4; ${ }^{1} \mathrm{H}$ NMR (400 MHz, Pyridine-d $\left.\mathrm{d}_{5}\right): \delta 8.58$ (d, $\left.1 \mathrm{H}, J=7.3 \mathrm{~Hz}\right), 6.86$ (d, 1H, $\left.J=2.0 \mathrm{~Hz}\right)$, $6.59(\mathrm{~d}, 1 \mathrm{H}, J=2.0 \mathrm{~Hz}), 5.76(\mathrm{~m}, 1 \mathrm{H}), 5.45(\mathrm{~m}, 1 \mathrm{H}), 5.20(\mathrm{dd}, 1 \mathrm{H}, J=12.4,3.9 \mathrm{~Hz}), 5.14-5.08(\mathrm{~m}, 2 \mathrm{H})$, $4.88(\mathrm{~m}, 1 \mathrm{H}), 3.53$ (dq, 1H, $J=13.6,3.5 \mathrm{~Hz}), 2.91(\mathrm{~m}, 1 \mathrm{H}), 2.68(\mathrm{~m}, 1 \mathrm{H}), 2.59-2.35(\mathrm{~m}, 4 \mathrm{H}), 2.21-2.15$ (m, 2H), 1.99-1.68 (m, 11H), 1.62-1.56 (m, 3H), 1.48-1.41 (m, 4H), 1.34-1.22 (m, 26H), 0.91 (t, 3H, J $=7.0 \mathrm{~Hz}) ; \mathrm{MS}(\mathrm{ESI}) \mathrm{m} / \mathrm{z}$ : Calcd for $\left(\mathrm{M}^{+}+\mathrm{Na}\right)$ 1031.33. Found: 1031.71 . 